\title{
ON THE INDEPENDENCE OF THE FIRST AND SECOND MATRICES OF AN ALGEBRA*
}

\author{
BY C. C. MACDUFFEE
}

1. Introduction. It is well known $\dagger$ that every linear associative algebra with a principal unit (modulus) is isomorphic with the algebra of its first matrices, and also with the algebra of its transposed second matrices. If the algebra has no principal unit, it can be represented as a matric algebra of $(n+1)$ th order matrices.

The condition that the algebra have a principal unit is not, however, necessary in order that the algebra be isomorphic with the algebra of its first or second matrices, as can readily be seen from examples. In this paper necessary and sufficient conditions for this isomorphism are obtained.

2. The Correspondence of Poincarê. Consider a linear associative algebra $\mathfrak{A}$ over a field $\mathfrak{F}$ with $n$ basal numbers $e_{1}$, $e_{2}, \cdots, e_{n}$, the constants of multiplication being $c_{i j k}$. Let us denote by $R_{i}$ the matrix $\ddagger\left(c_{i s r}\right)$, and by $S_{i}$ the matrix $\left(c_{r i s}\right)$, where $r$ determines the row and $s$ the column in which an element stands.

The conditions for associativity in $\mathfrak{A}$ may be written $\S$

$$
\sum_{k} c_{i k r} c_{j s k}=\sum_{k} c_{i j k} c_{k s r}, \quad(i, j, r, s=1,2, \cdots, n) .
$$

If we form the matrices in which the respective members of the above equation stand in the $r$ th row and $s$ th column, we have

$$
R_{i} R_{j}=\sum_{k} c_{i j k} R_{k}
$$

* Presented to the Society, Chicago, March 30, 1929.

$\dagger$ L. E. Dickson, Algebras and their Arithmetics, Chicago, 1923, p. 96.

$\ddagger R_{\boldsymbol{i}}$ and $S_{\boldsymbol{i}}$ are the first and transposed second matrices, respectively, of $e_{i}$. Dickson, loc. cit., p. 95.

$\S$ Dickson, loc. cit., p. 92. 
We may also write conditions (1) in the form

$$
\sum_{k} c_{r i k} c_{k j s}=\sum_{k} c_{i j k} c_{r k s}
$$

whence it follows that

$$
S_{i} S_{j}=\sum_{k} c_{i j k} S_{k}
$$

Thus both $R_{i}$ and $S_{i}$ are isomorphic under multiplication with the basal numbers $e_{i}$.

Every number $a$ of $\mathfrak{A}$ can be written

$$
a=a_{1} e_{1}+a_{2} e_{2}+\cdots+a_{n} e_{n},
$$

where the $a_{i}$ are in $\mathfrak{F}$. We define the first matrix $R(a)$ of $a$ by the equation

$$
R(a)=a_{1} R_{1}+a_{2} R_{2}+\cdots+a_{n} R_{n},
$$

and the second matrix $S(a)$ by

$$
S(a)=a_{1} S_{1}+a_{2} S_{2}+\cdots+a_{n} S_{n} .
$$

Thus the algebra $\mathfrak{A}$ is isomorphic with the algebra of matrices $R(a)$ if and only if $R_{1}, R_{2}, \cdots, R_{n}$ are linearly independent, and isomorphic with the algebra of matrices $S(a)$ if and only if $S_{1}, S_{2}, \cdots, S_{n}$ are linearly independent.

3. Two Invariants. If we apply to the basal numbers $e_{1}$, $e_{2}, \cdots, e_{n}$ the linear transformation

$$
e_{i}=\sum_{j} a_{i j} e_{j}^{\prime}, \quad a=\left|a_{r s}\right| \neq 0,
$$

with coefficients in $\mathfrak{F}$, the constants of multiplication are subject to the induced transformation*

$$
\sum_{i} c_{r s i} a_{i j}=\sum_{p, q} a_{r p} a_{s q} c_{p q j}^{\prime}, \quad(r, s, j=1,2, \cdots, n) .
$$

This may be written

$$
a c_{i s r}=\sum_{p, q, t} A_{r t} a_{i p} c_{p q t}^{\prime} a_{s q},
$$

* For example, see MacDuffee, Transactions of this Society, vol. 31 (1929), p. 81. 
where $A_{r t}$ denotes the cofactor of $a_{r t}$ in $A=\left(a_{r s}\right)$. Then

$$
R_{i}=\bar{A}^{-1}\left(\Sigma_{p} a_{i p} R_{p}^{\prime}\right) \bar{A}, \quad(i=1,2, \cdots, n),
$$

where $\bar{A}$ is the transpose of $A$.

If we denote by

$$
\sum_{j} k_{i j} R_{j}=0, \quad(i=1,2, \cdots, \rho),
$$

a maximal set of linearly independent linear relations among the $R_{j}$, we have

so that

$$
\bar{A}^{-1} \sum_{h, j} k_{i j} a_{j h} R_{h}^{\prime} \bar{A}=0
$$

$$
\sum_{h^{\prime}}\left(\sum_{j} k_{i j} a_{j h}\right) R_{h}^{\prime}=0, \quad(i=1,2, \cdots, \rho) .
$$

Since

$$
\left(\sum_{j} k_{r i} a_{j s}\right)=\left(k_{r s}\right) A
$$

and the matrix $\left(k_{r s}\right)$ of $\rho$ rows and $n$ columns is of rank $\rho$, we see that there are at least $\rho$ linearly independent linear relations among the matrices $R_{1}^{\prime}, R_{2}^{\prime}, \cdots, R_{n}^{\prime}$. Since (2) has an inverse, there are just $\rho$ such relations. Hence $\rho$ is invariant under transformation of coordinates.

Similarly we find that the number $\sigma$ of linearly independent linear relations among the matrices $S_{1}, S_{2}, \cdots, S_{n}$ is likewise invariant.

4. A Condition for the Independence of the Matrices. Suppose that exactly $\rho$ independent relations (5) hold among the matrices $R_{j}$. Form a matrix $B \equiv\left(b_{r s}\right)$ so that $b_{i j}=k_{i j}$ for $i=n-\rho+1, \cdots, n$, and take for the remaining $b_{i j}$ any convenient numbers of $\mathfrak{F}$ so that $B$ is non-singular. Apply a transformation (2) using $A=B^{-1}$. From (4) we have

$$
R_{i}^{\prime}=\bar{B}^{-1} \sum_{i} b_{i j} R_{j} \bar{B}, \quad(i=1,2, \cdots, n) .
$$


Hence $R_{n-\rho+1}^{\prime}=\cdots=R_{n}^{\prime}=0$ while $R_{1}^{\prime}, R_{2}^{\prime}, \cdots, R_{n-\rho}^{\prime}$ are linearly independent. We drop primes.

We now have

$$
c_{i j k}=0, \quad(i>n-\rho ; j, k=1,2, \cdots, n) .
$$

The associativity conditions (1) may be written

$$
\sum_{k=1}^{n} c_{i j k} c_{k s r}=\sum_{k=1}^{n} c_{i k r} c_{j s k}
$$

We consider only those equations in which $j>n-\rho$, and pass to matrices, obtaining

$$
\sum_{k=1}^{n-\rho} c_{i j k} R_{k}=0
$$

Since $R_{1}, R_{2}, \cdots, R_{n-\rho}$ are linearly independent,

$$
c_{i j k}=0, \quad(j>n-\rho ; k \leqq n-\rho ; i=1,2, \cdots, n) .
$$

Consider the linear set $\$$ composed of all numbers

$$
z=z_{n-\rho+1} e_{n-\rho+1}+\cdots+z_{n} e_{n} .
$$

We see readily from (6) and (7) that

$$
\mathfrak{B}=0, \quad \mathfrak{U} ß \leqq \Re,
$$

so that $\mathbb{B}$ is an invariant zero subalgebra of $\mathfrak{A}$ which has the additional property that $\mathfrak{B Y}=0$.

Conversely, let us suppose that $\mathfrak{A}$ has an invariant zero subalgebra $\mathscr{Z}$ of order $\rho$ such that $\mathscr{B Y}=0$. We take the basis numbers of $\nexists$ for $e_{n-\rho+1}, \cdots, e_{n}$ of a basis for $\mathfrak{A}$. Since $\mathfrak{Z} \mathfrak{U}$ $=0$, we have (7) and therefore $R_{n-\rho+1}=\cdots=R_{n}=0$.

Similar results hold for the second matrix.

THEOREM 1. A necessary and sufficient condition in order that there be exactly $\rho(\sigma)$ linearly independent linear relations among the matrices $R_{1}, R_{2}, \cdots, R_{n}\left(S_{1}, S_{2}, \cdots, S_{n}\right)$ is that $\mathfrak{Q}$ have an invariant zero subalgebra $\mathfrak{Z}(\mathfrak{W})$ of order $\rho(\sigma)$ such that $\mathfrak{Z Y}=0,(\mathfrak{Y} \mathfrak{W}=0)$, and no such subalgebra of order greater than $\rho(\sigma)$. 
It is known that in every algebra with a principal unit (including every semi-simple algebra) both the first and second matrices are independent.*

It can be seen directly that if $\mathfrak{A}$ is nilpotent or the direct sum of a nilpotent algebra and another algebra, the first matrices and also the second matrices are dependent. For we may choose normalized $\dagger$ basis numbers $e_{i}$ for the nilpotent algebra such that

$$
e_{i} e_{n}=e_{n} e_{i}=0, \quad(i=1,2, \cdots, n) .
$$

Hence $e_{n}$ serves both as $\mathbb{Z}$ and $\mathfrak{W}$ for Theorem 1 .

That $\rho$ is not necessarily equal to $\sigma$ is shown by the following example:

$$
e_{1}^{2}=e_{1}, \quad e_{1} e_{2}=0, \quad e_{2} e_{1}=e_{2}, \quad e_{2}^{2}=0 .
$$

We have

$$
R_{1}=\left(\begin{array}{ll}
1 & 0 \\
0 & 0
\end{array}\right), \quad R_{2}=\left(\begin{array}{ll}
0 & 0 \\
1 & 0
\end{array}\right), \quad S_{1}=\left(\begin{array}{ll}
1 & 0 \\
0 & 1
\end{array}\right), \quad S_{2}=\left(\begin{array}{ll}
0 & 0 \\
0 & 0
\end{array}\right) .
$$

Here $R_{1}$ and $R_{2}$ are linearly independent and give a representation of the algebra, while $S_{1}$ and $S_{2}$ are dependent. Thus $\rho=0, \sigma=1$, and $\mathfrak{B}$ is composed of multiples of $e_{2}$.

5. The Characteristic Equations. Let

$$
x=x_{1} e_{1}+x_{2} e_{2}+\cdots+x_{n} e_{n}
$$

be the general number of an algebra $\mathfrak{A}$, and define

$$
\delta(\omega)=|R(x)-\omega I|, \quad \delta^{\prime}(\omega) \equiv|S(x)-\omega I| .
$$

It is well known $\ddagger$ that $x \delta(x)=0$ and $x \delta^{\prime}(x)=0$, and if $\mathfrak{A}$ has a principal unit, also $\delta(x)=0$ and $\delta^{\prime}(x)=0$.

It is possible, however, for $\delta(x)=0$ or $\delta^{\prime}(x)=0$ even though $\mathfrak{A}$ has no principal unit.

THEOREM 2. A sufficient condition in order that $\delta(x)=0$ $\left(\delta^{\prime}(x)=0\right)$ is that $R_{1}, R_{2}, \cdots, R_{n}\left(S_{1}, S_{2}, \cdots, S_{n}\right)$ be linearly independent.

* Dickson, loc. cit., p. 95.

$\dagger$ Dickson, loc. cit., p. 176.

$\ddagger$ Dickson, loc. cit., p. 100. 
The proof is immediate, for by the Hamilton-Cayley theorem

$$
\delta(R(x))=0, \quad \delta^{\prime}(S(x))=0 .
$$

Since $\mathfrak{A}$ is isomorphic with the algebra of matrices $R(x)$ (or $S(x)$ ), we have $\delta(x)=0$ (or $\delta^{\prime}(x)=0$ ).

For the example of $\S 4$ we have

$$
\delta(\omega)=\omega^{2}-\omega x_{1}, \quad \delta^{\prime}(\omega)=\omega^{2}-2 \omega x_{1}+x_{1}{ }^{2} .
$$

Hence $\delta(x)=0$, while $\delta^{\prime}(x)=x_{1}^{2}-x_{1}^{2} e_{1}-x_{1} x_{2} e_{2}$.

Ohio State University

\section{ON THE NUMBER $\left(10^{23}-1\right) / 9$}

D. H. LEHMER

The purpose of this note is to save any further effort* in trying to factor the number $N=\left(10^{23}-1\right) / 9=111,11111$, $11111,11111,11111$ which in a previous paper was found to be composite. $\dagger$ This assertion was based on a negative result giving $3^{N-1} \neq 1(\bmod N)$.

On the basis of this conclusion Kraitchik $\ddagger$ attempted to factor $N$ arriving at another negative result that $N$ had no factors and therefore was a prime. This conflict of results led us to recompute the value of $3^{N-1}(\bmod N)$ which shows clearly a mistake in the original calculation arising from the choice of 3 for a base instead of another number prime to $10^{23}-1$. Such another base would have furnished the extra check which would have detected the error.

* A recent letter from Mr. R. E. Powers informs us that he has been to the trouble of finding 150 quadratic residues of $N$.

$\dagger$ This Bulletin, vol. 33 (1927), p. 338.

$\ddagger$ Mathesis, vol. 42 (1928), p. 386. 